\title{
Tres tratamientos psicoterapéuticos para pacientes con trastorno limite ("borderline") de la personalidad
}

\author{
Three treatments for borderline personality disorder
}

Clarkin J et al. Am J Psychiatry 2007; 164:922-928.

\section{Objetivo}

Comparar la eficacia de la terapia dialéctica conductual (TDC) la psicoterapia centrada en la transferencia (PCT) y la psicoterapia de apoyo (PA) en pacientes con trastorno "borderline" o límite de la personalidad (TLP).

\section{Diseño, lugar y pacientes}

Ensayo clínico aleatorizado y controlado sobre 90 adultos con TBP, realizado en New York, EEUU.

\section{Intervención}

Los pacientes fueron asignados en proporciones iguales a cada grupo y evaluados cada cuatro meses durante un año con evaluación de los diferentes síntomas a través de los instrumentos detallados entre paréntesis más abajo.

\section{Medición de resultados principales}

El objetivo principal fue tratar la intencionalidad suicida ("Overt agresión scale-modifield") la agresividad ("Anger, irritability and assault") y la impulsividad ("Barratt impulsiveness scale-ll"); y secundariamente la ansiedad ("Brief sympton inventory") la depresión ("Beck depresión inventory") y la adaptación social ("Global assessment of functioning scale" y "Social adjustment scale").

\begin{abstract}
Resultados principales
Se observaron mejorías en todos los dominios estudiados al cabo de un año de tratamiento, sin diferencias significativas entre los tres tratamientos investigados. Sin embargo, tanto la TCD como la PCT mejorarían la intencionalidad suicida por encima de la PA. Del mismo modo, la PCT y la PA mostrarían mejor desempeño para la ira. Respecto de los objetivos secundarios, los tres tratamientos mostraron mejorías en la mayoría de los síntomas evaluados, con un mejor desempeño para la PCT (mejoría en 10 de las 12 variables) respecto de la PA (seis de 12) y la TDC (cinco de 12).
\end{abstract}

\section{Conclusiones}

La PCT y la TDC serían los tratamientos indicados para pacientes con TLP que presenten intencionalidad o conductas suicidas. Palabras clave: trastorno límite de la personalidad, psicoterapia, suicidio. Fuente de financiamiento: Research Foundation.

\section{Comentario}

Los trastornos de personalidad en general y el TLP son un de los campos más complicados y difíciles de abordar dentro amplio abanico de los trastornos mentales ${ }^{1}$. La cuarta edición del Manual Diagnóstico y Estadístico de los Trastornos Mentales (DSM IV) señala una prevalencia del $2 \%$ en la población general, de $10 \%$ entres los pacientes ambulatorios y de $20 \%$ entre los pacientes internados ${ }^{2}$. Los fracasos en la adherencia y la pobre respuesta a los tratamientos convencionales -tanto psicológicos como farmacológicos- convierten a éste en un tema de acuciante interés para el campo clínico. Por lado, estos pacientes suelen consultar a sus médicos por problemáticas relacionadas; y por otro establecen un vínculo lábil y muchas veces tormentoso. Estas últimas características serían consecuencia de la activación de dos creencias básicas: la de "abuso" y la "abandono",,6.

La polisintomatología y la alta comorbilidad con otros trastornos mentales, la recurrencia de las crisis y el riesgo suicida muchas veces presente, unido al marcado déficit en el control de los impulsos que experimentan, junto al fracaso en las intervenciones estándar y a la sensación de desesperanza que generan en los equipos tratantes tratantes, convierten frecuentemente a estos pacientes en candidatos a internaciones psiquiátricas ${ }^{4}$. Por lo que se desprende la relevancia clínica de la presente investigación y la urgencia por buscar tratamientos que muestren eficacia clínica.

Es interesante observar que a pesar de la gran batería de evaluación utilizada y el tiempo de seguimiento, se han hallado modestos resultados en cuanto a la diferenciación de los tratamientos, lo que mostraría en principio la complejidad del cuadro psicopatológico. Un elemento importante de señalar es que no se han encontrado diferencias entre los grupos de pacientes estudiados sino entre los síntomas tratados.

\section{Conclusiones del comentador}

Más allá de las disputas entre los modelos teórico-clínicos que en todo campo existen, estas conclusiones confirman lo que los consensos de expertos ${ }^{3}$ y nuestra práctica clínica refuerza, en el sentido de tender a la integración -psicoterapéutica y farmacológica- de los tratamientos disponibles a nuestro alcance para tratar este trastorno. Entendiendo que, hasta el momento, ningún modelo de abordaje clínico (psicológico o farmacoterapéutico) por separado puede dar cuenta de la complejidad del cuadro clínico y de su polisintomatología.

Francisco Palacín [ Cátedra Clínica Psicológica, Psicoterapias, Emergencias e Interconsulta II. Facultad de Psicología. Universidad de Buenos Aires. Director de EQUIPO PSY, Adrogué. franciscopalacin@yahoo.com.ar ]

Palacín F. Tres tratamientos psicoterapéuticos para pacientes con trastorno límite ("borderline") de la personalidad. Evid Act Pract Ambul. 11(3): 75. May-Jun 2008. Comentado de: Clarkin, J; Levy, K; Lenzenweger, M. Evaluating three treatments for borderline personality disorder: a multiwave study. Am J Psychiatry 2007; 164:922-928. PMID: 17541052. Disponible en URL: http://www.ajp.psychiatryonline.org/cgi/reprint/164/6/922 (último acceso 27/06/08).

\section{Referencia}

1. American Psychiatric Association. (2000). Practice Guidelines for the treatment of psychiatric disorders, Washington, D.C.: American Psychological Association.

2. American Psychiatric Association.(1995). Manual Diagnostico y Estadístico de los Trastornos Mentales DSM-IV. Barcelona. Masson

3. Gunderson, J. (2001) Borderline Personality Disorder: A clinical Guide. American Psychiatric Press. Washington. DC.

4. Linehan, M. (1994). Cognitive Behavioral Treatment of Borderline Personality Disorder. New York. The Gilford Press.

5. Silva, D. Trastonos de la personalidad. Publicado en "Medicina Familiar y Practica Ambulatoria". Editores Rubinstein A, Terrasa S y col. 2da edición, Buenos Aires, Médica. Panamericana, 2006. pp 177-195.

6. Keegan, E. Abordaje cognitivo del trastorno límite de la personalidad. Escritos de psicoterapia cognitiva. EUDEBA.

7. Palacín, F. Modelo integrativo en psicoterapias de orientación cognitiva para los trastornos de personalidad límite. Ponencia de especialista. Consejo Superior del Colegio de Psicólogos de la Provincia de Buenos Aires. 